\title{
Brain-to-brain loop 2020: è ancora utile il ciclo di Lundberg?
}

\author{
Brain-to-brain loop: is the Lunberg concept useful in 2020?
}

\author{
Piero Cappelletti ${ }^{1}$ (D)
}

Ricevuto: 13 settembre 2017 / Accettato: 23 settembre 2017 / Pubblicato online: 13 novembre 2017

(C) Società Italiana di Patologia Clinica e Medicina di Laboratorio 2017

Riassunto Il più conosciuto schema di descrizione del Total Testing Process (TTP) e della generazione dell'informazione medica di laboratorio è il brain-to-brain loop di George Lundberg, originariamente descritto negli anni '80 del secolo scorso e da allora rivisto e riadattato più volte. Le modifiche più importanti sono legate all'introduzione dei concetti di pre-preanalitica/post-postanalitica, di colloquio tra cervelli clinica-laboratorio, di rete di cervelli in laboratorio e sul versante clinico, del ruolo del paziente e dell' outcome e all'integrazione con altre visioni riguardanti i modi, le caratteristiche e la semantica dello scambio informativo clinica-laboratorio. Lo schema è stato utilizzato principalmente come riferimento teorico per lo studio e classificazione degli errori e della qualità e dei suoi indicatori, ma anche per il management, la validazione e l'appropriatezza e altre caratteristiche specifiche del TTP. In generale, però, esso rappresenta la migliore sintesi di descrizione pratica e di visione teorica della generazione dell'informazione in Medicina di Laboratorio. Alla luce dei cambiamenti che sconvolgono la Medicina e la Medicina di Laboratorio sotto il profilo delle innovazioni tecnologiche e assistenziali, il concetto di brain-to-brain loop può mantenere la sua validità a condizione che una sua rivisitazione comprenda alcune caratteristiche: lo schema sia fuso con il concetto di interfaccia clinica-laboratorio amplificata, le fasi pre-pre e post-post siano pensate come momento del lavoro all'interfaccia di team multidisciplinari, venga descritto come colloquio tra reti di cervelli e il paziente sia efficacemente al centro del ciclo di generazione dell'informazione medica a lui dedicata.

$凶$ P. Cappelletti

pie.cappelletti@gmail.com

1 SIPMeL, Castelfranco Veneto, TV, Italia
Parole chiave Ciclo di Lundberg · Total Testing Process (TTP) · Interfaccia laboratorio-clinica $\cdot$ Medicina di Laboratorio

Summary The brain-to-brain loop, proposed by George Lundberg in the 80s, is the best-known description of the Total Testing Process (TTP) and of the generation of laboratory medicine information. Several integrations of the original scheme include the concepts of pre-preanalytical and post-postanalytical phases, conversation between clinical and laboratory brains, network of brains on both clinical and laboratory side, patients' role and their outcomes, and the fusion with other visions of Laboratory Medicine. It was utilized as theoretical reference for studying and classifying errors and quality indicators as well as laboratory management, verification/validation steps, and appropriateness of the whole TTP. Nevertheless, it is the best theoretical and practical synthesis of the laboratory generation of medical information, which is the essence of Laboratory Medicine. In nowadays changing times the concept could be still useful, if reviewed including a fusion with the concept of cliniclaboratory interface, the pre-pre and post-post phases are thought as work at the clinic-laboratory interface of multidisciplinary team, the conversation is between networks of brains, and it is effectively patient-centered.

Keywords Brain-to-brain loop · Total Testing Process $(T T P) \cdot$ Clinic-laboratory Interface $\cdot$ Laboratory Medicine

\section{Total Testing Process (TTP)}

Fornire informazioni per la salute degli esseri umani è la mission della Medicina di Laboratorio. Per rispondere adeguatamente alla sua natura, cioè "fornire informazioni cliniche", la Medicina di Laboratorio deve governare l'intero 
processo che la origina, attiva e utilizza [1]. Il punto chiave è l'interfaccia clinica-laboratorio [2], amplificata a dismisura dalla struttura a rete intra- ed extra-laboratorio, rivolta al clinico e al paziente, coinvolgente tutta l'equipe, caratterizzata dalla qualità della comunicazione e dalla sua coerenza con l'obiettivo clinico [1].

Il percorso di un esame di laboratorio dalla richiesta alla risposta e conseguente intervento medico è conosciuto con il termine americano di Total Testing Process (TTP), tratto dalla letteratura industriale e introdotto nella Medicina di Laboratorio probabilmente da Gambino [3] negli anni '70 del secolo scorso, ed è definito come "una serie di attività inter-relate e interagenti che trasformano un campione biologico del paziente in risultati analitici e informazioni diagnostiche".

\section{Brain-to-brain-loop}

Il più conosciuto schema di descrizione del TTP e della generazione dell'informazione medica di laboratorio è il brain-to-brain loop [4]. All'inizio degli anni '80 del secolo scorso, Georg Lundberg sentì il bisogno di rappresentare con un'immagine sintetica ed efficace un concetto apparentemente ovvio, il fatto cioè che le procedure di laboratorio sono finalizzate a una decisione clinica, e diede a questa icona il nome di "circuito dal cervello al cervello" del clinico. Descritto originariamente in 9 passi (richiesta, raccolta, identificazione, trasporto, preparazione, analisi, risposta, interpretazione, azione), è inteso come un "ciclo continuo" che individua i passaggi concettuali e materiali attraverso i quali la necessità del medico di sciogliere il quesito clinico del suo specifico paziente trova risposta nell'esame di laboratorio, eseguito su adeguato materiale e con una specifica richiesta attraverso l'esecuzione analitica e l'interpretazione dei risultati, cosicché possa essere iniziato un intervento appropriato al caso clinico in questione. Dieci anni dopo Lundberg presentò una versione aggiornata del suo schema [5], con una rilevante modifica: il primo passaggio (la richiesta dell'esame) è suddiviso in 3 passi e cioè la formulazione del problema clinico, la selezione del test e la richiesta vera e propria. Nella versione più matura, quindi, il loop comprende 11 passaggi: formulazione del problema clinico, selezione del test, richiesta, prelievo/raccolta, identificazione, trasporto, preparazione, analisi, risposta, interpretazione, azione. Burlina [6], che aveva introdotto il concetto in Italia a metà degli anni ' 80 del secolo scorso, commentava molto positivamente l'innovazione, ponendo l'accento sull'indissolubile legame tra le operazioni pre-analitiche e l'interpretazione dei risultati-l'altro pilastro della sua logica diagnostica - e sulla profonda aderenza della Medicina di Laboratorio italiana al concetto che tutte le fasi del TTP sono pienamente oggetto della disciplina.
Lo schema è stato utilizzato principalmente come riferimento teorico per lo studio e la classificazione degli errori e degli indicatori di qualità, in particolare da Plebani et al [7-11], ma anche per il management [12], la validazione [13] e l'appropriatezza [14, 15], per citare solo alcuni degli scopi e degli autori. In generale, però, ha rappresentato finora la migliore sintesi di descrizione pratica del TTP e di visione teorica della generazione dell'informazione medica di laboratorio [16], nonostante le critiche di eccessiva semplificazione e di inadeguatezza nella realtà attuale espresse da alcuni autori [17].

Lo schema ha subito, nel tempo, numerose integrazioni e rivisitazioni in rapporto ad alcuni temi predominanti: concetto di pre-preanalitica/post-postanalitica, seppure con significati differenti secondo i diversi autori [14, 18, 19]; colloquio tra cervelli clinica-laboratorio $[9,20]$ e concetto di rete di cervelli in Laboratorio [20] e sul versante clinico [21]; ruolo del paziente $[9,22,23]$ e importanza dell'outcome $[16,20,22,24]$; integrazione con altre visioni riguardanti la generazione dell'informazione di Laboratorio [18, 20].

\section{Le fasi pre-pre- e post-postanalitiche}

Secondo Hawkins [25], i diversi autori che agli inizi degli anni 2000 introducono il concetto delle fasi "pre-pre" e "post-post" analitiche hanno scopi diversi e sottendono definizioni differenti.

Nella Nexus vision di Goldschmidt [18] la classica tripartizione dell'iter diagnostico di laboratorio (fase preanalitica, analitica, postanalitica) viene estesa a 5 fasi, dove la iniziale pre-preanalitica richiede la descrizione del contesto del paziente e la valutazione della diagnostica di laboratorio comparata con altri strumenti diagnostici, mentre quella post-postanalitica dovrebbe contenere la descrizione del nuovo contesto del paziente, basato sull'integrazione delle nuove informazioni comunque ottenute, e una visione delle diverse interpretazioni e possibili conseguenze.

Laposata et al [19], viceversa, utilizzano il concetto per identificare le attività associate con l'iniziale selezione dei test e con l'interpretazione da parte dei clinici e per differenziarle dalle attività di raccolta e trasporto (fase preanalitica) e di reporting (fase postanalitica), in particolare collegandole alle nuove ricerche sugli errori in Laboratorio [7].

Più recentemente [11] le fasi pre-pre e post-post sono entrate come passaggi principali per definire i "cinque diritti" dei pazienti riguardo la qualità del TTP. Questa interessante visione ha l'obiettivo, da un lato, di mostrare la forte interrelazione delle diverse fasi tra loro e sull'esito del TTP e del paziente e, dall'altro, di superare il punto di vista "errori di laboratorio" e anche quello "qualità intrinseca" dello stesso per approdare a un più ampio punto di vista: quello del paziente e dei suoi diritti a una prestazione di alto valore. 
Fig. 1 Reinterpretazione dello schema di Lundberg. Il concetto del "ciclo" è fuso con quello di interfaccia clinica-laboratorio; il colloquio è tra due cervelli: clinico e laboratorio; il cervello del Laboratorio è un network di cervelli (l'equipe); le fasi pre-pre e post-post sono momenti di lavoro all'interfaccia; la focalizzazione al paziente e all'esito è messa in evidenza. La diapositiva, presentata nella relazione di apertura [20] del $21^{\circ}$ Congresso Nazionale SIMeL 2007, è stata pubblicata, con modifiche, nel 2010 in "Trattato Italiano di Medicina di Laboratorio" [16]

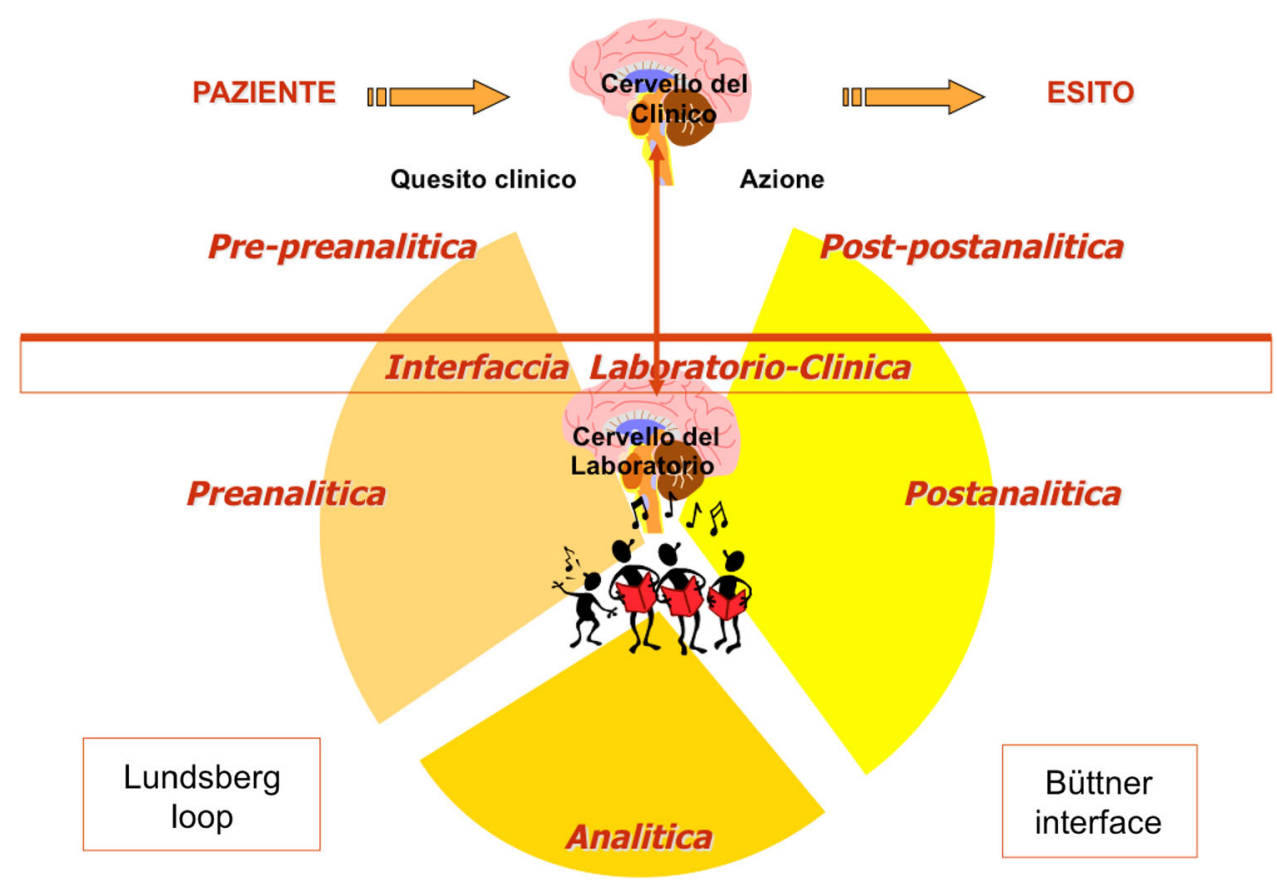

Tuttavia, nello specificare i diritti delle fasi pre-pre e postpost non si va oltre una definizione puramente fattuale delle stesse (identificazione del paziente e qualità del campione; interpretazione e documentazione). Anche il follow-up, citato nella fase post-post come "diritto" finale, si riferisce alla notifica e presa in carico del risultato non all'esito sul paziente.

Per altri [1], viceversa, lo schema di Lundberg serve come riferimento generale per l'interpretazione dell'essenza della Medicina di Laboratorio tra necessità diagnostica di un test e risposta del Laboratorio al quesito clinico. In particolare, per quanto riguarda l'appropriatezza, il concetto allora emergente [14] che essa in Medicina di Laboratorio non è un predicato della sola fase preanalitica (o pre-preanalitica) come selezione dei test ma di tutto il TTP, si basa sull'applicazione dello schema di Lundberg fuso con il concetto di interfaccia clinica-laboratorio di Büttner. In questo caso le fasi pre-pre e post-post sono il campo dell'attività generale di lavoro all'interfaccia, nei team multidisciplinari dedicati alle "evidenze", linee guida, protocolli di appropriatezza, feedback e audit.

\section{Il colloquio tra reti di cervelli}

Nel 2007 si introduce nello schema il colloquio tra cervelli (clinico e laboratorio) e il concetto di rete di cervelli (l'equipe) [20]. Nel modello originale, il "circuito" parte e si conclude nel cervello del medico curante e i diversi passaggi sono meramente "fattuali". Nell'impostazione italiana del 2007 [20] si rileva come in realtà il circuito si basi sul col- loquio tra due cervelli, quello del medico curante che origina la richiesta basata sul quesito clinico e applica la risposta al trattamento del paziente e quello del laboratorio che risponde al quesito clinico e che ha almeno due caratteristiche essenziali. Innanzitutto è posto all'interno del ciclo, a significare che deve governare il complesso delle fasi e non esclusivamente la fase analitica e, inoltre, è costituito da una rete di cervelli (team, equipe) necessaria per la pluralità e complessità delle azioni da svolgere lungo il TTP. Si tratta di un'attività coordinata e collaborativa di un'equipe complessa e articolata nelle sue responsabilità e autonomie, tenuta insieme dalla "mission" e dalla responsabilità clinico-organizzativa. Nel 2010 questa nuova visione [16] viene compiutamente descritta (Fig. 1), con la sottolineatura della finalità del brain-to-brain loop (outcome del paziente) e la necessaria fusione con altre visioni sulla generazione dell'informazione.

Nel frattempo si fa evidente che anche per il cervello del clinico non è più tempo di solipsismo: la complessità della medicina e dell'organizzazione sanitaria costruisce reti di cervelli (e di opere) a sostegno della cura e dell'assistenza, in particolare del paziente cronico. Nel 2007, Boone [21], nella sua riproposta dello schema di Lundberg, chiarisce come l'azione medica conseguente all'esecuzione del test lungo il TTP impatti non solo sul paziente ma sull'intera rete sociale (patient, family, community) e come sia sempre più evidente che l'azione terapeutica è frutto della rete di cervelli che struttura l'assistenza sanitaria [20].

Nella revisione del brain-to-brain concept 40 anni dopo, a cura di Plebani, Laposata e Lundberg [9], solo alcune di queste suggestioni vengono riprese, avendo la prevalenza l'utilizzo dello schema per la classificazione degli errori, 
intra- ed extra-laboratorio. Compare il colloquio tra cervello del medico e cervello del laboratorista, ma affrontati al punto di chiusura del ciclo, come uno scambio ristretto alla risposta (interpretazione?), senza allargarsi alle altre fasi e al governo del sistema. Del tutto assente, infine, una riflessione sulle "reti di cervelli" all'interno e all'esterno del Laboratorio, coinvolte nel TTP e nella cura del paziente.

\section{Il paziente e il suo outcome}

Nello schema originario di Lundberg l'attenzione è focalizzata alla sequenza di azioni prevalentemente laboratoristiche che, originate dal quesito clinico, determinano l'azione terapeutica e quindi presuppone ovviamente l'esistenza di un paziente, ma non ne esplicita il ruolo né lo coinvolge. $\mathrm{Nel}$ 1999 è lo stesso Lundberg [22], attento osservatore dell'evoluzione della realtà medica americana, a mettere in evidenza che finché un'azione sul paziente non è intrapresa a seguito dell'esame di laboratorio-fosse anche una motivata astensione dal trattamento-il ciclo dell'esame di laboratorio non è completato e che è il paziente il consumer dei test diagnostici e che quindi viene per primo. E aggiunge che: "We found in Los Angeles many years ago that the greater the extent to which the laboratory could control all steps in this process the greater likelihood the patient's interests would be served". Tuttavia il rapporto con il paziente è del medico curante e solo indirettamente del Laboratorio.

Nel 2005, Hickner et al [23] applicano lo schema di Lundberg alla medicina territoriale, con attenzione alla classificazione degli errori. Gli autori descrivono una fase preanalitica tradizionalmente centrata sulla selezione del test, ma nella fase postanalitica identificano passaggi in qualche modo nuovi: rispondere ai risultati e documentare la risposta, notificare i risultati al paziente e applicare i trattamenti conseguenti e seguire gli esiti nel tempo (outcome). Vi è un'interessante discussione sugli errori della fase di notifica dei risultati, che rivela il ruolo centrale dei valori e preferenze dei pazienti. Importante è la sottolineatura della necessità di seguire nel tempo l'effetto, seppure mediato, dei test sulla storia naturale del paziente. Si avverte in questa rilettura dello schema la diversa sensibilità del medico generale rispetto a quello ospedaliero.

Tuttavia nella revisione del brain-to-brain loop del 2011 [9] il coinvolgimento del paziente è ancora descritto quasi esterno a esso e comunque mediato dal cervello del clinico e vengono elencate numerose le preoccupazioni legate all'accesso diretto del paziente al Laboratorio. Vale anche qui, a mio parere, quanto scritto per il testo di Lundberg del 1999 [22]: il medico di laboratorio è al centro di una rete di rapporti tecnici e sociali in cui il paziente è essenziale; il Laboratorio deve tenere contatti diretti con il paziente per l'informazione preanalitica e analitica, per le attività diagnostiche ambulatoriali, per l'interpretazione della risposta e la consulenza; la centralità del paziente vale anche per la costruzione e l'attività del Laboratorio; la Medicina di Laboratorio ha un compito "sociale" di informazione/formazione dei pazienti e della società; è controproducente offrire un "patto" al paziente per poi escluderlo dalla consapevolezza del TTP che lo riguarda [1].

In effetti, il diretto rapporto con il paziente e il suo entourage non sfugge, come già accennato, a diversi autori [20, 21], ma è soprattutto Freedman [24] nel 2015 a riprendere e concretizzare il concetto della costruzione del TTP intorno al paziente e proporne un'illustrazione suggestiva, anche se poi non affronta il tema nel dettaglio. Fin dal titolo del suo lavoro, la Freedman [24] evidenzia il rapporto del TTP con l'outcome del paziente e la necessità di un lavoro di squadra finalizzato a questo scopo. Più recentemente [11], i "diritti" del paziente a un elevato valore dell'esame di laboratorio a lui dedicato danno vita a una nuova visione di un TTP rispettoso in ogni sua fase della qualità necessaria a un'efficace risposta clinica.

Nel 2014, anche Lundberg [26] sente la necessità di inserire esplicitamente l'outcome come $10^{\circ}$ passaggio del brainto-brain loop, in relazione non solo al contenimento dell'errore ma all'appropriatezza e alla necessità di aggiungere la verifica dell'esito legato all'esame.

\section{Visioni coordinate}

Lo schema di Lundberg è stato anche utilizzato come elemento essenziale di visioni globali della Medicina di Laboratorio e in particolare delle teorie riguardanti i modi, le caratteristiche e la semantica dello scambio informativo clinica-laboratorio. La Nexus vision [18], un'articolata descrizione della complessità della Medicina di Laboratorio, collaziona esplicitamente elaborazioni di Lundberg (brainto-brain loop), Truchaud (quality loop), Weggeman (knowledge feedback) e l'approccio GUM (Guidelines for Uncertainty of Measurements) in un modello coerente e comprensivo, basato sulle possibilità tecnologiche che consentono di digitalizzare l'ambiente diagnostico di un paziente finalizzato a supportare le responsabilità del Laboratoriouna risposta al quesito clinico nel contesto delle necessità del paziente-dove "contesto" significa che la qualità deve andare oltre "la risposta corretta al paziente giusto" per "assicurarsi che il paziente sia trattato correttamente".

Da parte di altri autori $[16,20]$ si pone l'accento sulla complementarietà del concetto di brain-to-brain loop e di interfaccia clinica-laboratorio e sulla necessità di una loro fusione. Dalle analisi teoretiche di Johannes Büttner in poi, l'attività essenziale del Laboratorio si attua mediante lo scambio di materiali e informazioni all'interfaccia, quasi sinciziale, tra Laboratorio e clinica. Ciò si traduce, in realtà, 
Fig. 2 Brain-to-brain loop 2020. La reinterpretazione dello schema contiene: la fusione con il concetto di interfaccia e la sua amplificazione dovuta all'organizzazione a rete della Medicina di Laboratorio; l'esplicitazione delle attività pre-pre e post-post come lavoro all'interfaccia di gruppi multidisciplinari; il dettaglio delle attività pre e post, intra- ed extra-laboratorio; le attività ad alto valore aggiunto: azioni proattive, suggerimenti diagnostici, consulenza, audit clinico; il colloquio tra reti di cervelli: laboratorio, team clinico, paziente; la centralità del paziente, dei suoi valori e preferenze e del suo outcome

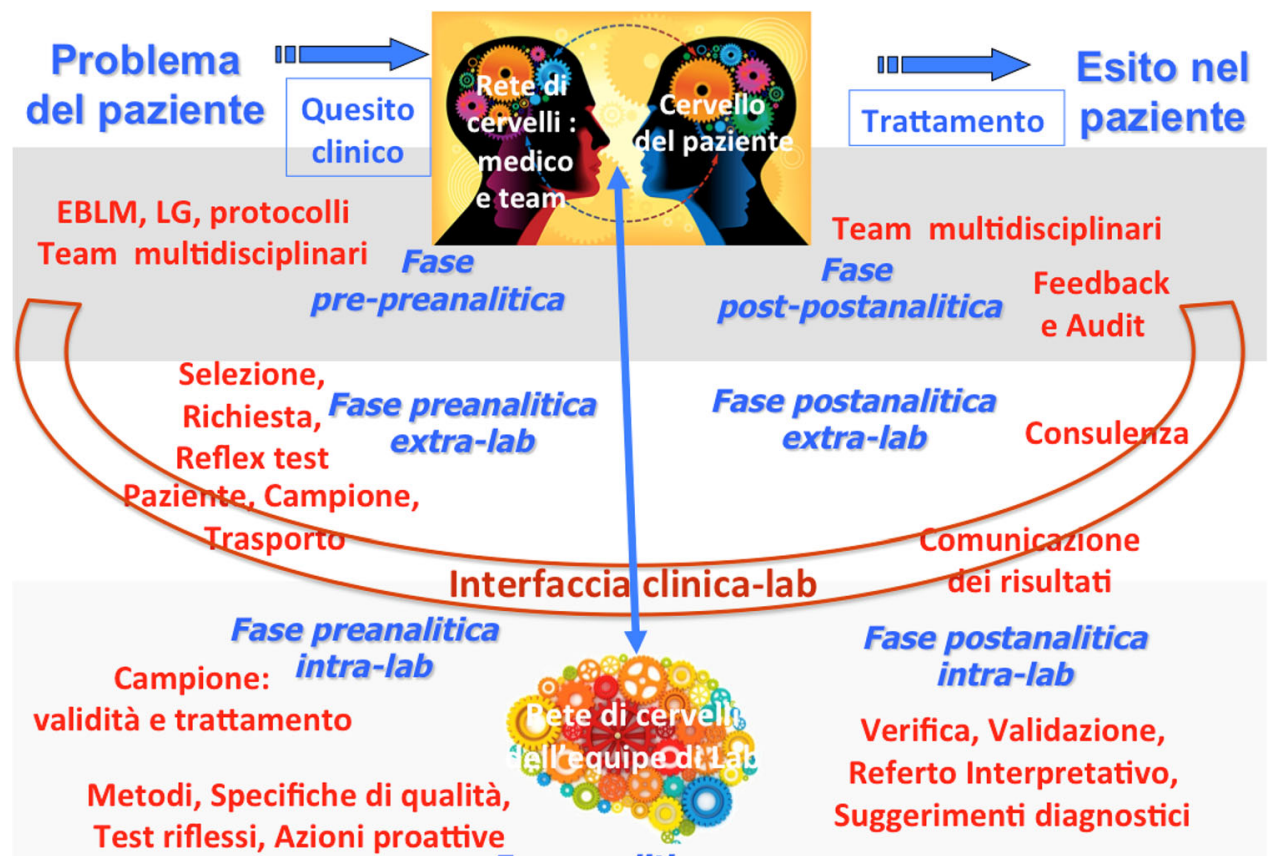

Fase analitica nella necessità della validazione di ogni passaggio del processo diagnostico-inteso come "brain-to-brain loop" ridescritto come colloquio tra due reti di cervelli (clinico e laboratorista) - a cura dell' attività coordinata e collaborativa di un'equipe complessa e articolata nelle sue responsabilità e autonomie, tenuta insieme dalla "mission" e dalla responsabilità clinico-organizzativa [1]. L'obbligatorietà per la Medicina di Laboratorio di impegnarsi in tutto il percorso del TTP, dalla formulazione del quesito clinico all'applicazione delle azioni conseguenti alle informazioni fornite, pertanto non esclusivamente nella fase analitica, ne è la necessaria conseguenza, oggi pienamente accettata dagli standard dei più avanzati sistemi di accreditamento/certificazione (ISO 15189:2012).

\section{Brain-to-brain loop 2020}

Il brain-to-brain loop di Lundberg mantiene ancora una sua validità come concettualizzazione teorico-pratica della Medicina di Laboratorio, alla luce dei cambiamenti che sconvolgono la Medicina e la Medicina di Laboratorio sotto il profilo delle innovazioni tecnologiche e assistenziali? Epner et al [17] non ne sono certi, in particolare per quel che attiene la descrizione dei punti più sensibili alla possibilità di errore. Tuttavia, gli esempi di dubbio che essi citano possono essere facilmente ricondotti allo schema: i send-out test (inviati ad altra sede) rientrano nella fase preanalitica del trasporto e postanalitica della trasmissione dei risultati; i reflex test rientrano nella fase preanalitica extra-lab della selezione dei test, nella fase analitica della scelta metodi/strumenti e della fase postanalitica del formato della risposta e sua interpretazione; gli add-on test rientrano nella fase analitica e postanalitica dei comportamenti proattivi. Sia i reflex test sia gli add-on test, di solito opportunamente concordati con i clinici, fanno parte delle attività pre-preanalitiche propedeutiche e generali del lavoro all'interfaccia dei gruppi multidisciplinari e possono far parte della fase post-postanalitica della verifica della loro validità ed effectiveness tramite feedback e audit. Gli extra-lab test, infine, sono riconducibili allo schema, come peraltro tentato da Hickner et al [23] ancora nel 2005, in particolare se si accetta il concetto dell'amplificazione dell'interfaccia clinica-laboratorio legata ai mutamenti dell'organizzazione delle cure.

Infatti, il ciclo di Lundberg può continuare a funzionare come visione teorico-pratica della generazione dell'informazione medica di laboratorio, anche per gli esami fuori dal Laboratorio (POCT) e dall'Ospedale (territorio, home), ad alcune condizioni: 1) lo schema sia fuso con il concetto di interfaccia clinica-laboratorio amplificata, come spiegato precedentemente; 2 ) le fasi pre-pre e post-post siano pensate non solo come la parte extra-lab delle fasi pre- e postanalitiche ma come momento del lavoro all'interfaccia (team multidisciplinari per "evidenze", linee guida, protocolli di appropriatezza, feedback e audit); 3) venga descritto come colloquio tra reti di cervelli (Laboratorio-team clinico e assistenziale-paziente); 4) il paziente sia effettivamente al centro del ciclo di generazione dell'informazione medica a lui dedicata (problema di salute-negoziazione su valori e preferenze-effectiveness).

La proposta che ne consegue di un brain-to-brain loop 2020 è illustrata nella Figura 2. Nella versione per la Medi- 
Fig. 3 Brain-to-brain loop 2020. Reinterpretazione dello schema per la Medicina extra-ospedaliera e il paziente ambulatoriale. Rispetto alla precedente si noti la migrazione di attività preanalitiche (preparazione del paziente, identificazione, prelievo e/o raccolta del campione e suo trasporto) dalla fase extra-lab alla fase intra-lab intesa come punto di prelievo e/o come attività ambulatoriale specifica $\mathrm{e}$ la diretta inter-relazione con il paziente sia in fase di informazione e gestione preanalitica, sia in fase analitica proattiva, sia in fase postanalitica di comunicazione dei risultati, consulenza e audit

Fig. 4 Brain-to-brain loop 2020. Reinterpretazione dello schema per le attività in Point-Of-Care Testing (POCT). La gran parte delle attività del Laboratorio non è rappresentata da operazioni esecutive intra-lab, ma d'impostazione pre-pre e di controllo post-post, di scelta di metodi/strumenti e specifiche di qualità, di formazione degli addetti e di gestione globale della qualità con aumento di lavoro all'interfaccia per tutta l'equipe

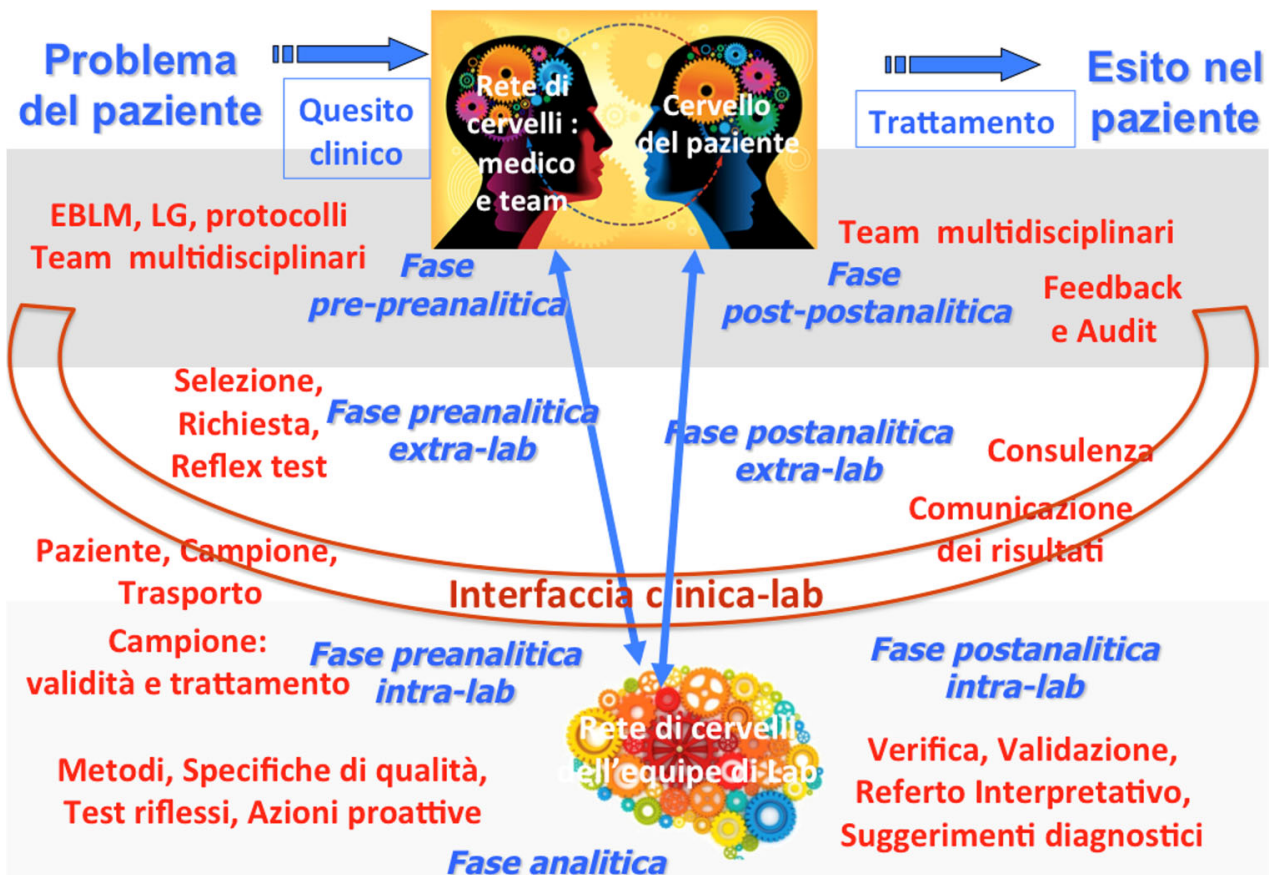

Fase analitica

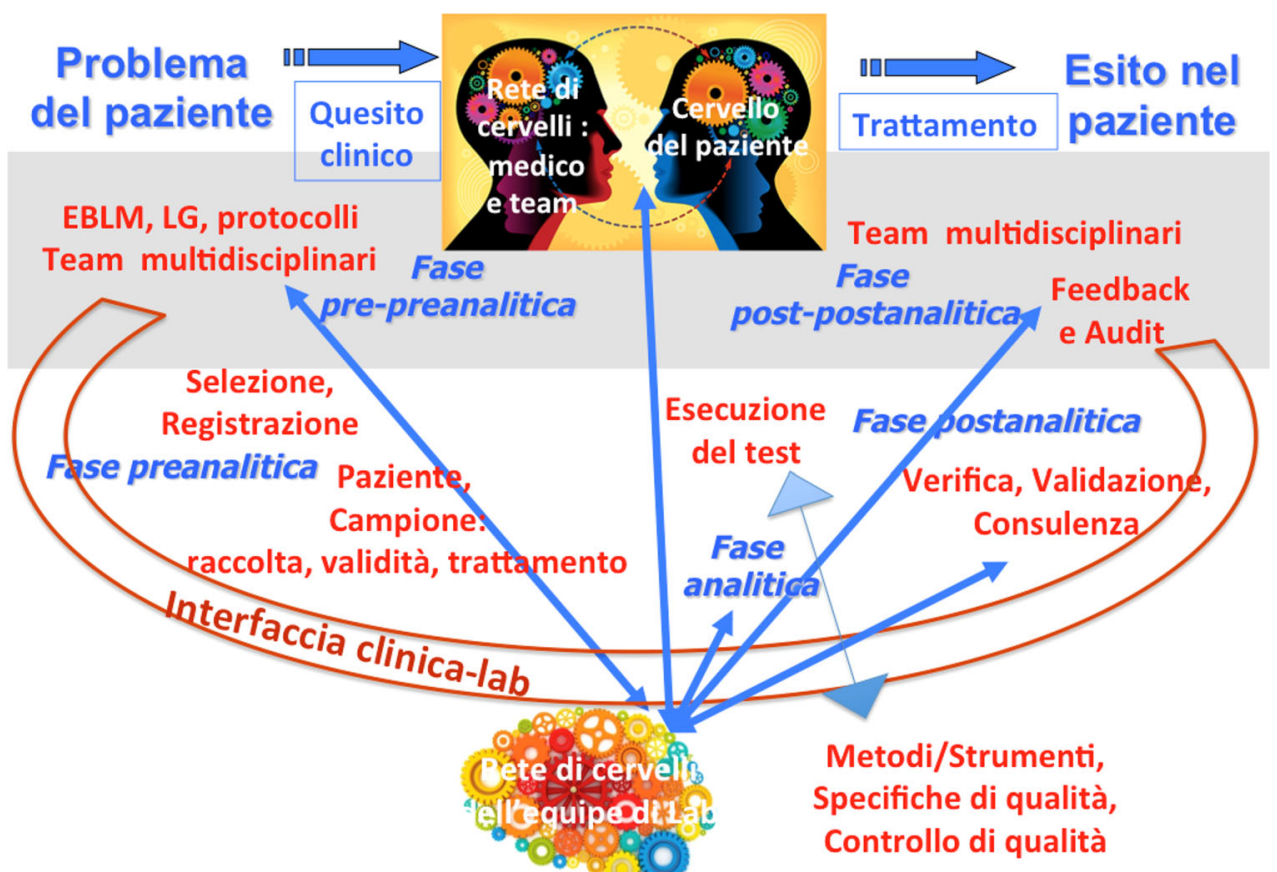

cina Generale (Fig. 3), la modifica più importante sta nella migrazione di attività preanalitiche dalla fase extra-lab (preparazione del paziente, identificazione, prelievo e/o raccolta del campione) alla fase intra-lab intesa come punto di prelievo/raccolta che deve essere annesso o sotto il controllo del Laboratorio e/o come attività ambulatoriale specifica. Altro aspetto importante è la diretta inter-relazione con il paziente sia in fase di informazione preanalitica, sia in fase analitica proattiva, sia in fase postanalitica di comunicazione dei ri- sultati e consulenza. Nella versione per POCT (Fig. 4), la gran parte delle attività del Laboratorio non è rappresentata da operazioni intra-lab, ma d'impostazione pre-pre e di controllo post-post, di formazione degli addetti, di scelta di metodi/strumenti e specifiche di qualità e di gestione globale della qualità (controllo di qualità interno ed esterno, allineamento e verifica strumentale, controlli su documentazione, verifica degli esiti). Aumenta pertanto il lavoro all'interfaccia di tutta l'equipe. 
Questa teorizzazione sufficientemente descrittiva seppure sintetica, infine, può essere assunta come riferimento per mostrare i luoghi, metaforici e non, in cui si manifestano i ruoli cui sono chiamati, oggi, i professionisti della Medicina di Laboratorio: sacerdoti della tecnologia, pianificatori e manager, consulenti clinici e attori del governo clinico [27].

\section{Conflitti di interesse Nessuno.}

Studi condotti su esseri umani e animali Per questo tipo di studio non è richiesto l'inserimento di alcuna dichiarazione relativa agli studi effettuati su esseri umani e animali.

\section{Bibliografia}

1. Cappelletti P (2005) La risposta della Medicina di Laboratorio al quesito clinico. RIMeL/IJLaM 3(Suppl 1):15-23

2. Büttner J (1997) Good laboratory practice: the medical aspects. Eur J Clin Chem Clin Biochem 35:251-256

3. Gambino SR (1970) Met and unmet needs of the automated clinical laboratory. Trans N Y Acad Sci 32:816-820

4. Lundberg GD (1981) Acting on significant laboratory results. JAMA 245:1762-1763

5. Lundberg GD (1990) Critical (panic) value notification: an established laboratory practice policy (parameter). JAMA 263:709

6. Burlina AB (1990) La tollerabilità dei farmaci, il laboratorio ed il "brain-to-brain loop". Progr Med Lab 3:137-138

7. Plebani M, Ceriotti F, Messeri G et al (2006) Laboratory network of excellence: enhancing patient safety and service effectiveness. Clin Chem Lab Med 44:150-160

8. Plebani M (2009) Exploring the iceberg of errors in laboratory medicine. Clin Chem Acta 404:16-23

9. Plebani M, Laposata M, Lundberg GD (2011) The brain-to-brain concept for laboratory testing 40 years after its introduction. Am J Clin Pathol 136:829-833

10. Plebani M, Sciacovelli L, Aita A (2017) Quality indicators for the Total Testing Process. Clin Lab Med 37:187-205

11. Plebani M (2016) Towards a new paradigm in laboratory medicine: the five rights. Clin Chem Lab Med 54:1881-1891
12. Barr JT, Silver S (1994) The total testing process and its implications for laboratory administration and education. Clin Lab Manage Rev 8:526-542

13. Giavarina D (2004) L'appropriatezza e l'EBM. Riv Med Lab-JLM 5(Suppl):64-67

14. Cappelletti P (2006) Appropriatezza in Ematologia di Laboratorio. RIMeL-IJLaM 2:119-129

15. Cappelletti P (2016) Appropriateness of diagnostics tests. Int $\mathbf{J}$ Lab Hematol 38(Suppl 1):91-99

16. Cappelletti P (2010) Medicina di Laboratorio. In: Plebani M, Galzigna L (eds) Trattato Italiano di Medicina di Laboratorio. Biochimica Clinica e Generale, vol I. Piccin, Padova, pp 9-104

17. Epner PL, Gans JE, Graber ML (2013) When diagnostics testing leads to harm: a new outcomes-based approach for laboratory medicine. BMJ Qual Saf 22(Suppl 2):ii6-ii10

18. Goldschimdt HMJ (2004) The NEXUS vision: an alternative to the reference value concept. Clin Chem Lab Med 42:868-873

19. Laposata M, Dighe A (2007) "Pre-pre" and "post-post" analytical error: high-incidence patient safety hazards involving the clinical laboratory. Clin Chem Lab Med 45:712-719

20. Cappelletti P (2007) La Medicina di Laboratorio e il miglioramento delle cure: un servizio essenziale, una prospettiva interdisciplinare, una visione olistica. RIMeL/IJLaM 3(Suppl):19-24

21. Boone $\mathbf{J}$ (2007) Presentation at the Institute on Critical Issues in health laboratory practice: managing for better health. September, 23-26, Atlanta, Centers for Disease Control and Prevention

22. Lundberg GD (1999) How clinicians should use the diagnostic laboratory in a changing medical world. Clin Chim Acta 280:3-11

23. Hickner JM, Fernald DH, Harris DM et al (2005) Issues and initiatives in the testing process in primary care physician offices. Jt Comm J Qual Patient Saf 31:81-89

24. Freedman DB (2015) Towards better test utilization-strategies to improve physician ordering and their impact on patient outcomes. EJIFCC 26:15-30

25. Hawkins R (2012) Managing the pre- and post-analytical phases of the total testing process. Ann Lab Med 32:5-16

26. Lundberg GD (2014) Adding outcome as the 10th step in the brain-to-brain laboratory test loop. Am J Clin Pathol 141:767-769

27. Cappelletti P (2012) Scenari e ruoli della Medicina di Laboratorio ai tempi della crisi: da Modena a Salerno ed oltre. Riv Ital Med Lab 8:183-189 